\title{
Air Quality Monitoring System Based on ISO/IEC/IEEE 21451 Standards
}

\author{
K. S. E. Phala, A. Kumar, and Gerhard P. Hancke, Senior Member, IEEE
}

\begin{abstract}
An air quality monitoring system (AQMS) based on IEEE/ISO/IEC 21451 standards is presented. In the development of AQMS, we have used the GSM wireless communication module. The developed system is capable of real-time measurement of air polluted gases such as $\mathrm{CO}_{2}, \mathrm{CO}$, $\mathrm{NO}_{2}$, and $\mathrm{SO}_{2}$. The machine-to-machine communication of the air quality monitoring station and $\mathrm{PC}$ with the sink node was successfully implemented. Various gas sensor technologies were evaluated for the system and ultimately electrochemical and infrared sensors were used. Hardware and software for an AQMS was designed and implemented. The AQMS uses an array of sensors to take measurements of the ambient air surrounding it and wirelessly transmits the data to the base station. A graphical user interface (GUI), which makes it easy for end user(s) to interact with the system, was developed. Gas concentration values are plotted on the GUI. The defined calibration of the instruments at time interims assures that the desired accuracy is sustained. This paper offers further elucidations to design disputes raised in earlier studies.
\end{abstract}

Index Terms-Air quality monitoring, GSM module, sensor array, graphical user interface, ISO/IEC/IEEE 21451standards.

\section{INTRODUCTION}

$\mathbf{O}$ ver the past half century, there has been an increasing number of natural disasters such as heavy rainstorms, monsoons, global warming, debris flow, etc. These cause rising the concentration of greenhouse gases in the earths troposphere region [1]. Greenhouse gases are also emitted from fossil fuel used to burn and clear tropical forests [2]. The level of greenhouse gases brings severe threats to man's life and results in the drastic increase in the number of cases of lung cancer, asthma, coronary artery infection, pneumonia, chronic bronchitis, and other pulmonary infection, etc. [3]. Most of the developed countries have been developing air quality and weather (temperature and humidity) monitoring stations in the populated areas or outside the city for preventing the effects of air quality.

K. S. E. Phala is with the Department of Electrical, Electronic and Computer Engineering, University of Pretoria, Pretoria 0002, South Africa (e-mail: ksephala@gmail.com).

A. Kumar is with the Department of Electrical, Electronic and Computer Engineering, University of Pretoria, Pretoria 0002, South Africa and also with the CSIR-CBRI, Roorkee, India (e-mail: anuj.kumar@up.ac.za; anujkumar@cbri.res.in).

G. P. Hancke is with the Department of Computer Science, City University of Hong Kong, Hong Kong, and also with the University of Pretoria, Pretoria 0002, South Africa (e-mail: gp.hancke@ cityu.edu.hk).
Most of these air quality monitoring stations have been using primitive methods of monitoring air quality, such as collecting air samples and analysing the samples in laboratories. These methods are reliable but inefficient and expensive. Basically, analytical instruments such as spectrometers and gas chromatographs may be used but they suffer from the same disadvantages as the primitive methods and they also require skilled professionals to operate them. We need a comprehensive approach for the real-time monitoring of air quality to reduce the impacts of climate change. According to this, the monitoring of greenhouse gases in air is becoming significant in the context of quality of life [4], [5]. In recent years, smart sensor-actuator networks have taken in a dynamic role in the extension of instruments and we have recently embarked on using smart sensors and standards for the measurement of air quality [6], [7]. The environment monitoring systems mainly consist of three parts such as monitoring nodes, coordinating nodes with a smart devices (PC/smart phone) and some kind of actuators [8], [9]. A monitoring processor of environmental air pollution parameters such as $\mathrm{O}_{3}, \mathrm{CO}, \mathrm{NO}_{2}$, and $\mathrm{SO}_{2}$ was reported in [10]-[12]. The monitoring processor is based on a gas sensor array, signal processing of the sensor, and a sensor-actuator interface capability. They used the semiconductor sensor array. The gas discrimination of the air-conditioned system for the measurement of air quality was proposed in [13]. They explained the casting of the data through the web. Jelicic et al. presented a method of monitoring of air quality parameters with the help of a wireless sensor network and infrared gas sensor. They also explained techniques for decreasing the power consumption in the sensor, network and node [14]. Chen et al. developed a smart phone based volatile organic compound monitoring system. They also proposed a single integrated wireless device for monitoring the volatile organic compound [15]. Chung et al. presented a method of remote monitoring of room environment with a wireless sensor network. They also interface a personal digital assistant (PDA) and PC [16]. Kan et al. developed a wireless sensor network (WSN) based environment monitoring system (EMS). GPS module is used in the development of system. The GPS system with WSN are provided the information in the location in the remote areas and they focussed on power consumption, mobile communication, and immovability of data transmission [17]. Kumar et al. developed a low cost comfort sensing system for built environment. They proposed the real-time monitoring and control the air quality and thermal comfort parameter through air conditioning and mechanical ventilation [18]. Ekuakille et al. proposed a cognitive wireless sensor network approach in the monitoring of volatile organic compounds and also used the semiconductor sensor array [19]. The power reduction technique node and communication level are explored in 
[20]. A GPRS sensor array based air pollution monitoring technique was reported in [21]. It is worth mentioning that much work is required to commercialize such a system. A wireless sensor network based real-time monitoring of toxic compounds in the environment was reported in [22], [23]. The design and operation of a data acquisition system for solar radiation remote monitoring and analysis was presented in [24]. Most of the above existing systems are suffer from the several drawbacks and thus amendments are unceasingly being pursued. These issues includes, amongst others, high platform cost, interference of the gas sensing module, enhanced accuracy, portability, easy to operate, data storage capacity of the server, data storage capacity in the sensing node, real-time monitoring commercial system, improved noise immunity, and most importantly the issue of data transmission, and machine-to-machine communication. When designing for low cost, portability, automatic data transmission, data storage at the server, machine-to-machine communication, a multidisciplinary approach is necessary.

In this paper, an air quality monitoring system for ambient air monitoring in compliance with ISO/IEC/IEEE 21451 standards is presented. It has several progressive features such as easy to operate, low cost, fast response time with scientifically acceptable accuracy. The system is suitable for national air monitoring networks, urban air monitoring networks, industrial pollution monitoring, roadside air monitoring, environmental impact assessments, short term hot spot, sport campus, play ground or stadium pollution monitoring, etc.

\section{ISO/IEC/IEEE 21451 FAMILY}

ISO/IEC/IEEE 21451 standard is series of standards like as ISO/IEC/IEEE $21451-1,2,4, \& 7$ to be inter-operable. They have been explore the implementation criteria of transducer independent interface (TIM) in the devices and also proposed the format criteria of the transducer electronic data sheet (TEDS). They also explore the commands for reading and writing the data in the system, and controlling of the TIM. Application programming interfaces (APIs) are defined to facilitate communications between the TIM and objects (system). ISO/IEC/IEEE 21451-1 defines a smart transducer object model (STOM) and communication methods to facilitate the access of smart transducers (ST) in a network. ISO/IEC/IEEE 21451-2 defines serial interfaces for connecting transducer modules to a network. ISO/IEC/IEEE 21451-4 defines a mixed-mode transducer interface that allows the transfer of digital transducer electronic data sheet and analogue sensor signals on the same wires. The details of IEEE/ISO/IEC 21451 standard information was presented in [25].

\section{SYSTEM OVERVIEW}

The air quality monitoring system was divided into two parts; firstly, the remote air quality base station and secondly, a sink node with PC. The remote air quality base stations take air quality measurements and wirelessly transmits the data via a sink node to the server, which is typically a PC or laptop computer. GSM modules are used for the wireless communication between the base stations and sink node. Fig. 1 represents the developed air quality monitoring systems. The developed air quality monitoring (AQM) system can be used for the real-time measurement of the quality of greenhouse gases such as $\mathrm{CO}_{2}, \mathrm{CO}, \mathrm{NO}_{2}$, and $\mathrm{SO}_{2}$.

Both subsystems are broken down further and implemented independently of each other. A single supply design was chosen for the remote air quality base station hardware system. Using a single supply eliminates the need for negative voltages and also reduces the power consumed by the sensor node. Fig. 2 and Fig. 3 represents the hardware infrastructure of the remote AQM station and photograph respectively. The AQM station is powered by a battery. The battery voltage is regulated to produce a constant $5 \mathrm{~V}$. Two regulators are used, one to power the GSM module and the other to power the rest of the sensor array.

\section{A. Sensor Array}

The sensing unit is a group of sensor known as a sensor array [7], [26]. There are five types of gas sensor available in the market, i.e. electrochemical, infrared, semiconductor, catalytic bead, and photo-ionization. We have selected the sensor based on the performance parameters such as accuracy and low power consumption. In the development of AQM station, the electrochemical and infrared gas sensors performed better compared to others. These sensors are also very selective to the target gas.

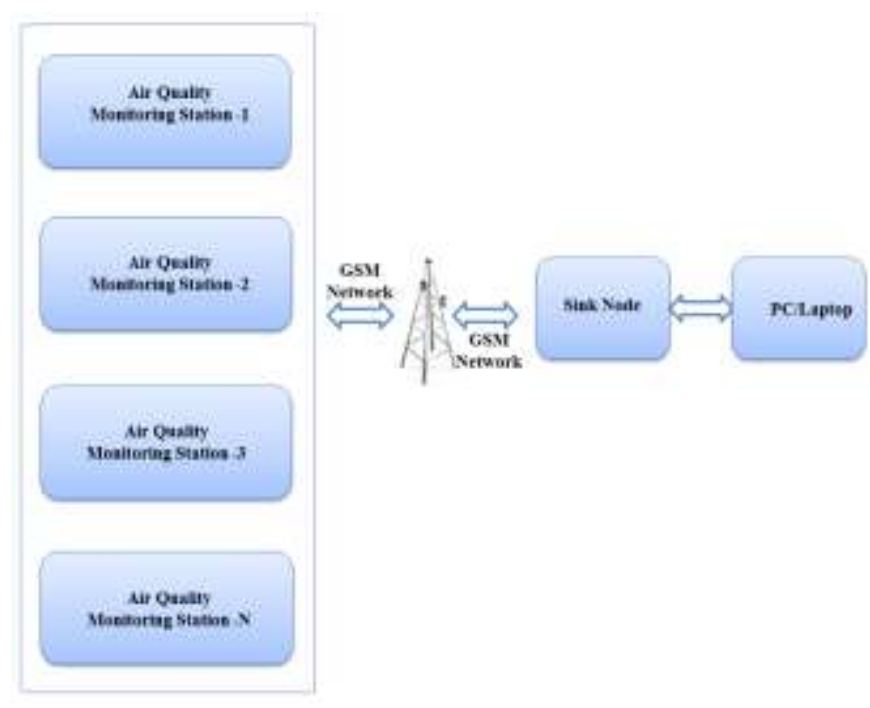

Fig. 1. System structure of AQMS

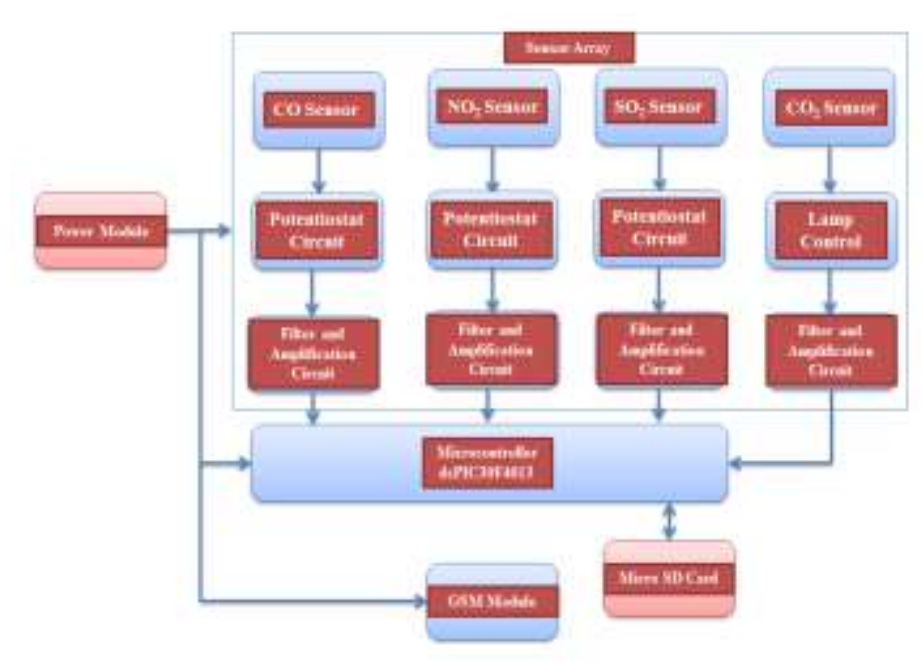

Fig. 2. Hardware infrastructure diagram of air quality monitoring station. 


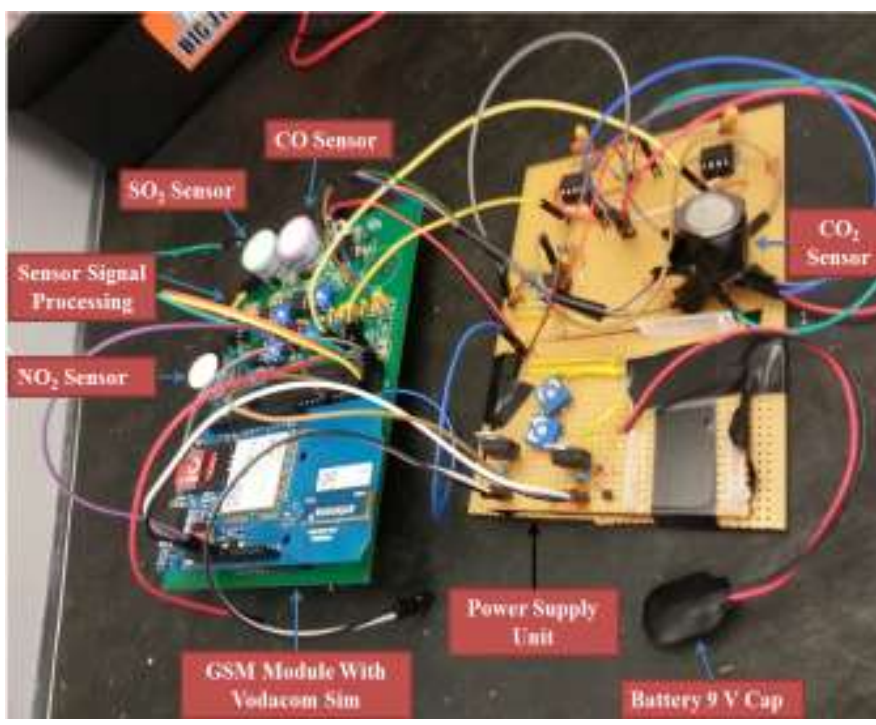

Fig. 3. Photograph of the air quality monitoring station.

The characteristics of the sensors used are summarised in Table I.

Table I

Sensor used in the development of AQM station

\begin{tabular}{|l|l|l|l|l|}
\hline \multicolumn{1}{|c|}{ Sensor } & $\begin{array}{c}\text { Target } \\
\text { Gas }\end{array}$ & $\begin{array}{c}\text { Range } \\
(\mathbf{p p m})\end{array}$ & $\begin{array}{c}\text { Resolution } \\
(\mathbf{p p m})\end{array}$ & $\begin{array}{c}\text { Response } \\
\text { Time } \\
(\mathbf{s e c o n d s})\end{array}$ \\
\hline EC4-500-CO & $\mathrm{CO}$ & $0-500$ & 1 & $<30$ \\
\hline $\mathrm{EC} 4-20-\mathrm{SO}_{2}$ & $\mathrm{SO}_{2}$ & $0-20$ & 0.1 & $<35$ \\
\hline EC4-20-NO $\mathrm{NO}_{2}$ & $\mathrm{NO}_{2}$ & $0-20$ & 0.1 & $<35$ \\
\hline IR11BD & $\mathrm{CO}_{2}$ & $0-3000$ & 100 & $<20$ \\
\hline
\end{tabular}

\section{1) Electrochemical $\mathrm{CO}, \mathrm{SO}_{2}$, and $\mathrm{NO}_{2}$ Sensors}

We use the three electrochemical sensors for $\mathrm{CO}, \mathrm{SO}_{2}$, and $\mathrm{NO}_{2}$ from $S G X$ Sensor Tech and they all use the same signal conditioning circuitry with only the gain adjusted for the different measuring ranges and the different sensor sensitivities. The designed circuit for the sensors is shown in Fig. 4. The electrochemical sensor's advantages, and disadvantages were reported in [9] and [10].

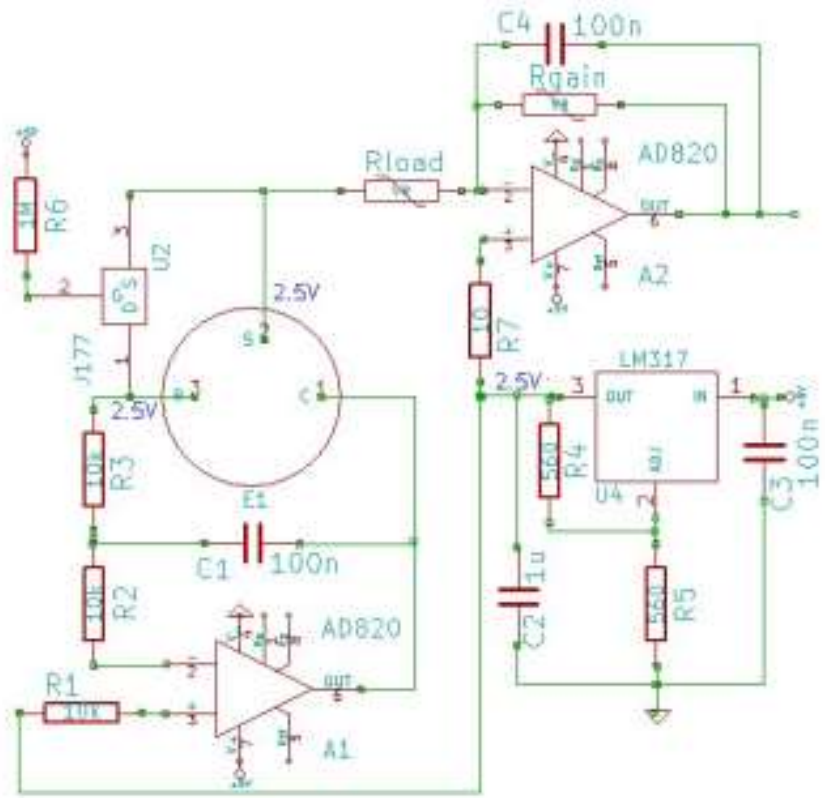

Fig. 4. Signal conditioning circuitry for electrochemical sensors.
As the gas concentration changes the potential at the sensing electrode $(S)$ changes due to the chemical reactions taking place on its surface. This can lead to incorrect measurements being taken. A basic potentiostatic circuit is used to keep the sensing electrode at a constant potential with respect to the reference electrode $(\mathrm{R})$. We have used the amplifier (A1) in the potentiostatic circuit mode. The Op-amp (A1) balance the current required by the sensing electrode and to provide the current to the counter electrode (C). The output from U4 is the input to the non-inverting terminal of $\mathrm{A} 1$ to provide the offset. This input is compared to the input on the inverting terminal from the reference electrode and the difference is amplified and fed into the counter electrode. The feedback configuration of the potentiostatic circuit makes the reference and sensing electrodes follow the input voltage from U4 very closely. Ideally no current should flow from the reference electrode but this is never the case so A1 is selected to have a very small input bias current, less than $5 \mathrm{nA}$. The AD820 from Analog Devices which was used has a typical input bias current of $2 \mathrm{pA}$. The improved single supply design requires the ground node to be moved to 2.5 $\mathrm{V}$ which means the voltage at the reference must also be change to follow this voltage.

The resistors $R_{2}$ and $R_{3}$ protect $A 1$ from static high voltage shocks when the input is switched on. Their values are chosen to be high so that they limit the amount of current being drawn from the reference electrode. $R_{1}$ and $C_{1}$ are included for additional stability and noise reduction. The potential between the reference and sensing electrodes need to be kept equal even when the power is off. This is so that when the power is turned on the sensors stabilise quickly otherwise it will take several hours for the sensors to settle. A shorting FET transistor (J177) was used for this. When the power is on, the transistor act as an open circuit and A1 is responsible for keeping the sensing electrode at the same potential as the reference electrode. When the power is off; the transistor acts as a short circuit connecting the sensing and reference electrodes and providing a zero bias voltage. The sensor's output current is converted to a voltage using a transimpedance amplifier. The sensor output current is very low and operational amplifier A2 is used to amplify the sensor output current. In the amplification process, the OPAMP A2 is used in transimpedance configuration.

$\mathrm{R}_{\text {gain }}$ determines the gain by which the transimpedance amplifier amplifies the sensor current. $R_{\text {gain }}$ was used to adjust the range and sensitivity of the sensors to meet the specifications of the system. The internal sensor resistance plus load resistor $\left(\mathrm{R}_{\text {load }}\right)$ and internal sensor capacitance to form an $\mathrm{RC}$ circuit. If a large value is selected for $\mathrm{R}_{\text {load}}$, noise is rapidly reduced but the response time increases. If a small value for $R_{\text {load }}$ is selected, the response time goes down but the noise increases. Thus the choice of $R_{\text {load }}$ is a trade-off between fast response time and best noise performance. Variable resistors are used for $\mathrm{R}_{\text {gain }}$ and $\mathrm{R}_{\text {load }}$ to add flexibility to the system. The outputs of the sensors drift over time and so $\mathrm{R}_{\text {gain }}$ and $\mathrm{R}_{\text {load }}$ can be adjusted to keep constant outputs. When different sensors are used in the system, $\mathrm{R}_{\text {gain }}$ and $\mathrm{R}_{\text {load }}$ can be adjusted for the range and sensitivity of those sensors. The non-inverting terminal of A2 is connected to the output of $\mathrm{U} 4$ through a small resistor $\mathrm{R}_{7}$ to be obtained the required $2.5 \mathrm{~V}$ offset. The correlation between sensor output voltage $\left(\mathrm{V}_{\text {out }}\right)$ and gas concentration (C) can be expressed as equation 1 


$$
\mathrm{C}(\mathrm{ppm})=\mathrm{P}_{\mathrm{f}} \cdot \mathrm{V}_{\text {out }}
$$

Where $\mathrm{P}_{\mathrm{f}}$ is the proportionality factor.

The gas sensing range, power consumption, gain, and proportionality factor of the developed module are given in Table II.

Table II

Developed sensor module parameters

\begin{tabular}{|c|c|c|c|c|}
\hline $\begin{array}{c}\text { Sensor } \\
\text { Module }\end{array}$ & $\begin{array}{c}\text { Measuring } \\
\text { Range }\end{array}$ & $\begin{array}{c}\text { Proportionality } \\
\text { Factor }\left(\mathrm{P}_{\mathrm{f}}\right)\end{array}$ & Gain & $\begin{array}{c}\text { Power } \\
\text { consumption }\end{array}$ \\
\hline $\mathrm{CO}$ & $\begin{array}{c}0.5 \mathrm{ppm}- \\
20 \mathrm{ppm}\end{array}$ & 4.1 & 48 & $9.0936 \mathrm{~mW}$ \\
\hline $\mathrm{SO}_{2}$ & $\begin{array}{c}0.04 \mathrm{ppm}- \\
2 \mathrm{ppm}\end{array}$ & 0.55 & 40 & $0.72 \mathrm{~mW}$ \\
\hline $\mathrm{NO}_{2}$ & $\begin{array}{c}0.01 \mathrm{ppm}- \\
0.5 \mathrm{ppm}\end{array}$ & 0.1046 & 33 & $11.2712 \mathrm{~mW}$ \\
\hline
\end{tabular}

\section{2) Infrared $\mathrm{CO}_{2}$ Sensor}

$\mathrm{CO}_{2}$ is measured using a nondispersive infrared sensor (or NDIR sensor). There are few alternative technologies available for measuring $\mathrm{CO}_{2}$ in the part per million (ppm) ranges. A lamp within the sensor is a source of infrared radiation. The radiation is passed through two channels and is detected on the other side using pyro-detectors. One channel is a reference channel, which passes through the IR radiation without altering it. The other channel is the active or sensing channel. The target gas absorbs the IR radiation in the active channel effectively attenuating it. Two pyro detectors are used to detect the radiation passing thorough the active and reference channels. The output of the pyro detectors is compared and the difference is related to the gas concentration. The output from the pyro detectors is very small and must be amplified and filtered to remove any noise. The outputs can then be sampled using a microcontroller. The peak-to-peak of the outputs must be determined in order to calculate the gas concentration. The sensor used is the IR11BD from SGX Sensor Tech. Fig. 5 shows the designed circuit for the infrared sensor.

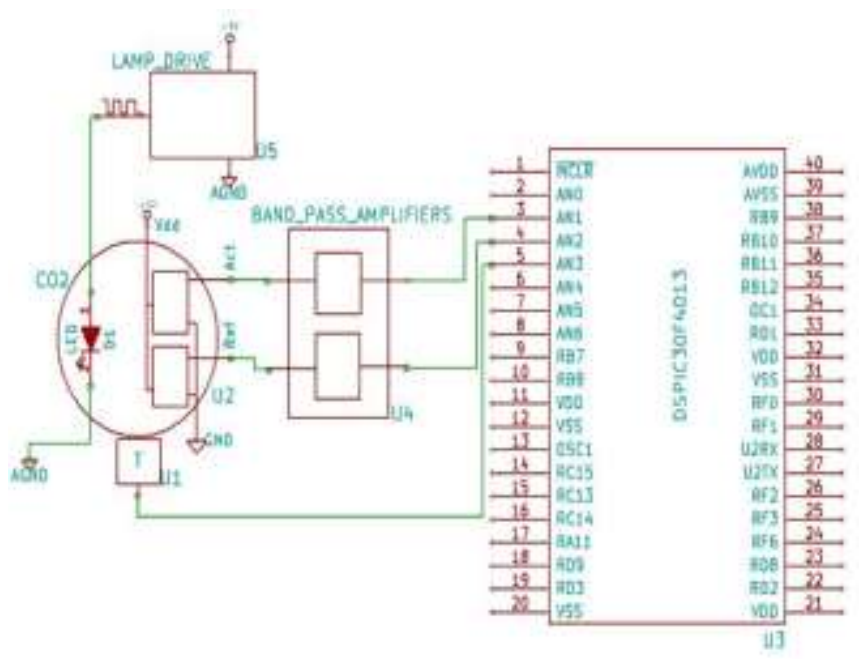

Fig. 5. Circuit diagram of the $\mathrm{CO}_{2}$ infrared sensor.

A 555 timer is used to generate a $4 \mathrm{~Hz} 50 \%$ duty cycle square wave that drives the internal lamp of the sensor. The timer is powered by a separate $9 \mathrm{~V}$ battery in order to isolate the transient currents from the lamp, and can generate the desired wave with a peak-to-peak value equal to its supply voltage. The high peak-to-peak value is required for the sensor to function correctly. The output of the sensor is affected by temperature. As the temperature changes, so does the pyro outputs. The nonlinear behaviour is compensated by attaching a temperature sensor to the infrared sensor and sampling the temperature together with the infrared sensor outputs. The compensation is then done in software from the temperature readings. An LM35 temperature sensor was used. The sensor has a very good accuracy and is pre-calibrated. The outputs from the pyro detectors are put through band pass amplifiers to amplify their small outputs and allows only the desired $4 \mathrm{~Hz}$ signal to pass through. The active and reference band pass amplifiers should be identical to produce accurate results. Fig. $6(a$ and $b$ ) shows the response of the active band pass amplifier, which looks similar to the reference band pass amplifier. The outputs are then sampled using a MCU and a peak detection algorithm is used to determine their peak-topeak values. Temperature compensation is then done on the peak-to-peak values. When the sensor is used for the first time a calibration procedure is done to determine the ZERO and SPAN. The value of the ZERO is set in the clean air (absence of $\mathrm{CO}_{2}$ gas) and it is the ratio of the peak-to-peak value of the active detector to the peak -to-peak value of the reference detector.

$$
Z E R O=\frac{A C T_{\circ}}{R E F_{\circ}}
$$

where $A C T_{o}$ is the peak-to-peak value of the active detector and $R E F_{o}$ is the peak-to-peak value of the reference detector. The SPAN is the proportion of radiation that impinges on the active element of the detector that has the ability to be absorbed by the target gas (known concentration of the target gas). It is given by the equation 3

$$
S P A N=\frac{\left(1-\frac{A C T}{Z E R O \times R E F}\right)}{\left(1-e^{\left(-a C^{n}\right)}\right)}
$$

where ACT is the current peak-to-peak of the active detector, REF is the current peak-to-peak of the reference, a $=2.49$ and $n=0.811$ are linearization constants found in the sensor data sheet [27] and $\mathrm{C}$ is the gas concentration in \% vol. The temperature compensation is added to the SPAN using the equation 4

$$
S P A N(C O M P)=S P A N+\left[\beta \times\left(\frac{T-T_{C A L}}{T_{C A L}}\right)\right]
$$

where $\beta(=0.084)$ is a constant and value found in the [27], $\mathrm{T}_{\mathrm{CAL}}$ is the temperature at calibration and $\mathrm{T}$ is the current temperature. When the calibration is done the calibration values are stored to be used to calculate the gas concentration at a later stage. The peak-to-peak values are normalised before they are used in the calculations of the gas concentration.

$$
\text { Normalized }_{\text {ratio }}=\frac{A C T}{Z E R O \times R E F}
$$

The gas concentration (\%) is then calculated using the equation 6

$$
\bar{C}=\left(\frac{-\ln \left[1-\left(\left(1-\text { Normalised }_{\text {ratio }}\right) / \operatorname{Span}(\operatorname{comp})\right)\right]}{a}\right)\left(\frac{1}{n}\right)
$$

The concentration of $\mathrm{CO}_{2}$ in \% volume. The equation 7 is used to convert it into ppm.

$$
C(p p m)=10000 \times \bar{C}
$$



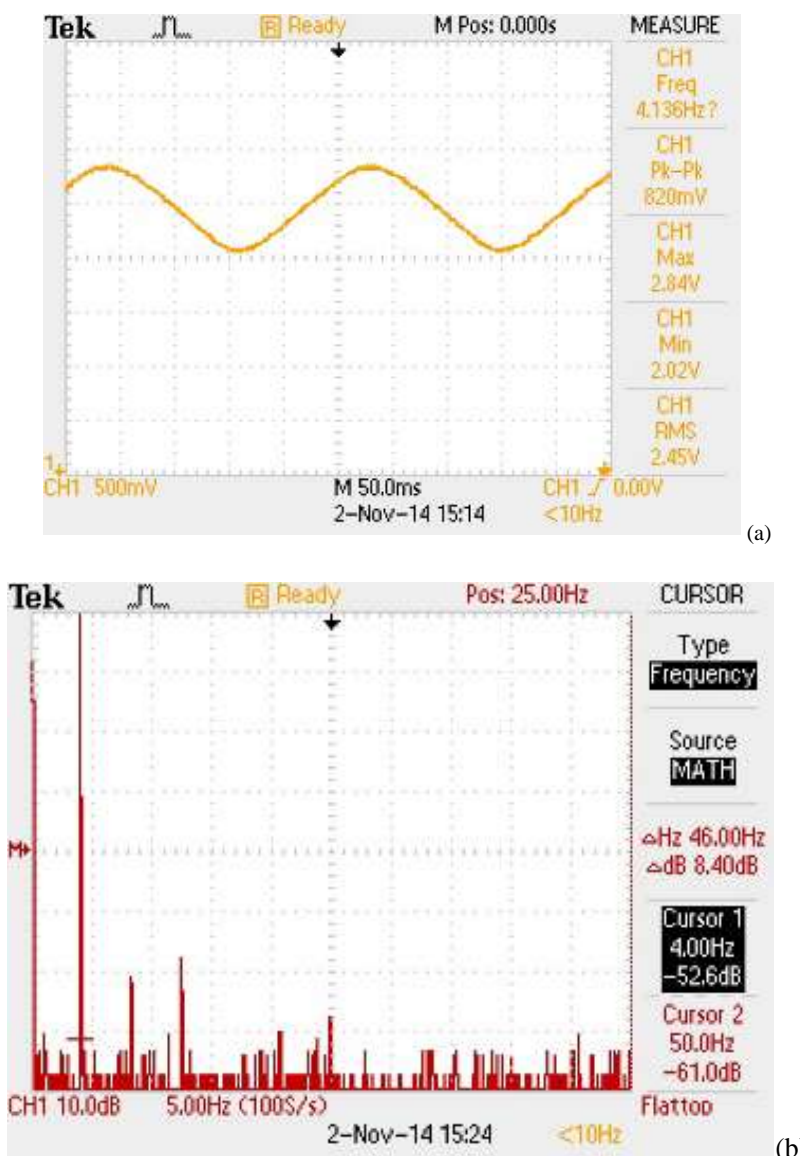

Fig. $6(\mathrm{a} \& \mathrm{~b})$. Time and frequency response of the active band pass amplifier.

The measuring gas sensing range of the developed $\mathrm{CO}_{2}$ sensor module is lies between 100ppm to 2000ppm. The power consumption was observed to be $180 \mathrm{~mW}$.

\section{B. Microcontroller}

The dsPIC30F4013 MCU was selected to perform all the processing on the sensor node. It is a 16-bit MCU with a modified Harvard architecture. It has a wide operating voltage $(2.5 \mathrm{~V}$ to $5.5 \mathrm{~V})$, which made it ideal for the system. Its 12-bit ADC satisfies the resolution requirements of the system and the 13 input channels are more than enough. The MCU is programmed using $\mathrm{C}$ programming language. It is set up to automatically sample all the signals from the sensors and interrupts when done. The gas concentration is then calculated from the sampled data. The MCU communicates serially with the GSM module using UART. The gas concentration data is sent to the GSM where it is transmitted wirelessly. The advantage and disadvantage of the dsPIC30F4013 microcontroller were reported in [28].

\section{Wireless Communication}

The remote sensor node communicates with the base station wirelessly. The communication is unidirectional with only the air quality monitoring station transmitting data. GSM modems are used for the wireless communication. Subscriber identity module (SIM) cards are inserted into the GSM modems that enable them to connect to cellular networks. Vodacom SIM cards were selected to be used but any SIM cards can work. In South Africa; GSM operates in the $900 \mathrm{MHz}$ and $1.8 \mathrm{GHz}$ bands. Vodacom networks cover a wider area of South Africa and they are more reliable than most service providers. The sensor node is able to transmit from anywhere in South Africa where Vodacom networks are available. Different protocols for the wireless communication were considered. A TCP/IP connection with the base station as the server and the AQMS as the client would have been the most efficient method. Unfortunately, it is not possible to obtain static IP addresses in South Africa. The IP address of the SIM card changes every time the modem is switched off. The use of short message service (SMS) was decided on. Using SMS's incurs additional cost to the system. There are a lot of different GSM modules available that are developed by different manufacturers. These all have different interfaces and power requirements. The Arduino GSM shield was chosen as the modem to be used. The module uses standard AT commands making it easy to communicate with the device. It can operate from two voltage levels ( 3 or 5 volts), which meets the power requirements of the system. The module is fitted with an onboard antenna. The SIM card slot on the module makes it easy to insert and remove the SIM card. The module is small, compact and relatively cheap. The advantage, command, specification, and disadvantage of the GSM Ardunio module were reported in [29].

The data transmission from one electronic device to other electronic device is based on communication technology known as Machine-to-Machine (M2M) communication. $\mathrm{M} 2 \mathrm{M}$ is the key technology of the data transfer between sensors/actuators and smart devices/PC. The M2M communication technologies are wireless, Bluetooth, cellular, ZigBee, WiFi, WiMax, generic DSL (xDSL), wireless LAN (WLAN), and fiber to the $\mathrm{x}$ (FTTx). The applications of $\mathrm{M} 2 \mathrm{M}$ communication are in different areas such as telemedicine (health care), intelligent building and management, environment, supply chain management, automotive, traffic control, military, warehouse management, robotics, logistic services, etc. The literature of M2M communications are available but limited. Data aggregation, processing, machine-to-machine communication and actuation for cyber-physical system were proposed in [30]. The wireless technologies and challenging aspects in $\mathrm{M} 2 \mathrm{M}$ communications were reported in [31] and they also explained the recently used technologies such as Bluetooth (IEEE802.15.1), ZigBee (IEEE802.15.4) and WiFi (IEEE802.11b) in different environment [31]. The issues in the M2M communication in 3GPP networks were reported in [32]-[35]. Dujak et al explained the smart meter and M2M service enablement node based energy consumption approach in smart buildings. Energy consumption profile are displayed graphically [36]. Nikaein et al. proposed an M2M system architecture based on LTE/LTE-A and they also explained the delays associated with each part of the system. They analysed the three real-time M2M applications such as intelligent transport system, gaming machine, and smart environment [37], [38]. The cellular based machine-tomachine communication with $\mathrm{P}-2-\mathrm{P}$ protocol stack was reported in [39] and [40]. Hasan et al. discussed the M2M communication techniques for cellular networks. They also discussed the overload control mechanisms to evade congestion caused by machine type communication devices [41]. Lee et al. discussed the solution of congestion problem in M2M systems. Moreover, they have explained that the $\mathrm{CR}$ (Cellular) type of M2M communication is superior to conventional M2M communication [42]. Kruger et al. proposed the Internet of Things vision in an industrial wireless sensor networks environment. They also explained the advantage and disadvantages based on Internet of Things 
systems [43]. Shaban et al. proposed an urban air quality monitoring system with forecasting models [44].

In this article, we have developed the communication between GSM module to GSM Module and the communication with the server machines through TCP/IP connection. The results are displayed on the GUI apps running on computer or smart devices.

\section{Graphical User Interface}

A sophisticated, yet easy to use graphical user interface was designed. The graphical user interface (GUI) software was developed using MATLAB and it is capable of performing a variety of functionalities. When the software is started the user must select the USB port where the sink node is connected. The GUI will then test communication with the GSM by sending some commands in the background. Once communication is established, the GUI is ready to receive data from the remote sensor node. It is not known before hand when the sensor node will transmit the data and so a call back function, which is essentially an interrupt in MATLAB, was implemented. When the GSM receives an SMS, it sends a notification to the GUI that triggers the call back function. The GUI then sends AT commands for reading SMSs to the GSM and the GSM returns the message content of the SMS. The data is then processed and displayed. The gas concentration values are plotted, each on its own axis. The temperature reading and battery voltage reading are displayed on edit boxes. Fig. 7 shows a screenshot of the GUI. The data being received is also stored in a data text file. The user can click on plot saved data to retrieve and plot the stored data. This can be useful when monitoring gas concentration for long periods of time.

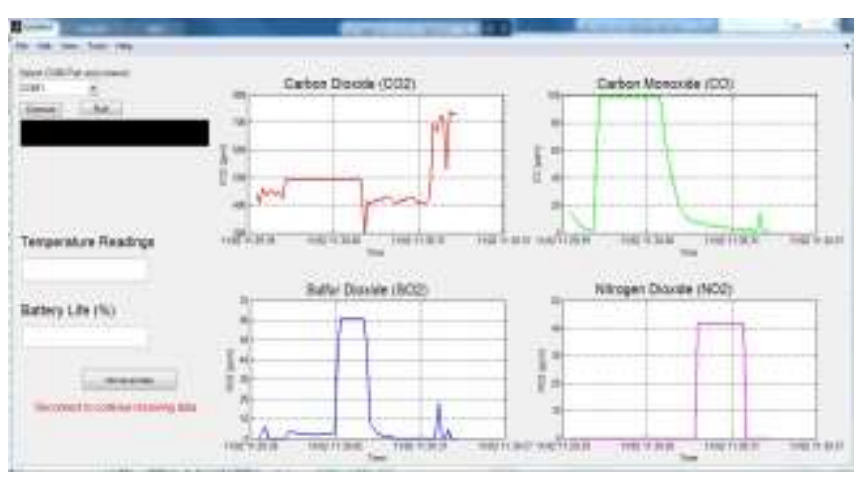

Fig. 7. Graphical user interface for clients.

\section{E. Calibration of the sensor Module}

Calibration is a vigorous issue in the designing of instruments for reliability. The calibration of the instruments is required at regular time intervals for maintaining the desired accuracy. Basically, the wireless communication based instrument calibration study is an embryonic stage [9], [10]. We have completed the calibration process into three steps.

\section{1) Calibration of the developed sensor module}

The calibration of the developed sensor module was described in detail in [11].

\section{2) Calibration of the developed entire (AQMS) system}

In the calibration process of the complete system, we have designed the sink node with GSM. The sink node is connected to Laptop/PC through USB. At the base station the data is captured by the sink or receiving node, which is connected to a computer running the GUI software. The sink node serially forwards the data to the computer. The real time data is displayed on a GUI-PC. An incubator is used for calibrating and testing of the developed system. The incubator is simply a rectangular plastic box with a hole on the side to fit a pipe that is used to pump in gas. The developed sensor node is powered on and placed inside the incubator that is then closed. The known gas cylinders were used to pump the gas into the incubator and we have repeated the process of section E-1 point (i) and (iii). The sensor node then takes measurements of the gas concentration. The calibration setup on real-time testing of the module and machine-to-machine communication are shown in Fig. 8 (a and b). Table III represents the accuracy of the developed system as per existing system.

Table III

Accuracy of the Measured Parameter

\begin{tabular}{|c|c|c|}
\hline Measured Gas & Instrument with Company Name & Accuracy \\
\hline $\mathrm{CO}$ & IAQ-Calc ${ }^{\mathrm{TM}}$ IAQ 7545 Track Plus & $\pm 2 \%$ \\
\hline $\mathrm{CO}_{2}$ & Trak Plus Q-8552 \& *554 & $\pm 4 \%$ \\
\hline $\mathrm{SO}_{2}$ & GAXT-S-DL (BW-Technology) & $\pm 3 \%$ \\
\hline $\mathrm{NO}_{2}$ & GAXT-D (BW - Technology) & $\pm 4 \%$ \\
\hline
\end{tabular}

\section{3) Control of cross sensitivity or interfering gases}

The process of controlling of cross sensitivity or interfering gases are based on two methods, such as, first is applying a bias voltage, and second is shorting the JFET. The control of cross sensitivity or interfering gases were explained in details in [11].

The sensitivity (nA/ppm) of the electrochemical sensor is changed corresponding to temperatures $0.1-0.3 \%$ per Kelvin and the humidity are not affected directly [9], [10]. The relative humidity between $15 \%-90 \%$ is not affected to the output of the electrochemical sensors. The experimental study shows that relative humidity below $15 \%$ or above $90 \%$ affected to the performance of the sensor. This affect of humidity in the electrochemical sensor is due on electrolyte of the cell. This process ensues very intentionally and depends upon the temperature, of the electrolyte. We have reckoning temperature and humidity effects on sensors and has been resolved these issue through calibration process based on data sheet.

\section{RESUlTS}

An air quality monitoring system (AQMS) based on wireless sensor network was designed, built and tested. The system was divided into two subsystems; a base station and a remote air quality station. The remote air quality station takes air quality measurements and wirelessly transmits them to the base station where they are processed and presented to the end user(s) through a GUI. The subsystems were designed and implemented independently of each other. The various subsystems of the air quality monitoring system were evaluated in order to determine the accuracy of the system. Fig. 9 shows a bar graph comparison of the performance of the different electrochemical sensors. $\mathrm{CO}_{2}$ was also measured successfully using the infrared sensor.

The graphical user interface (GUI) has been implemented successfully and Fig. 10 represents the on-line data updating on GUI based on real-time measurement for greenhouse gases. No loss of data occurs when the data packets are sent from the mobile phone until they are plotted on the graphical user interface. 
The measurements received are also successfully being appended to the data file. No loss of data occurs when writing to or reading from the data file.

\section{CONCLUSION}

In this paper, we have presented an air quality monitoring system (AQMS) based on the IEEE/ISO/IEC 21451 standard. The developed system was tested in a laboratory environment (in-situ environment). The prototype system consists of the air quality monitoring station, communication links, a sink node module and a data server. We have developed the GSM module based sink node with data server PC. The real-time data are saved in a micro SD card in text format and also saved in the data server (PC). For the data base we have chosen MySQL as the DBMS. Electrochemical and infrared sensors were used to measure the concentrations of $\mathrm{CO}, \mathrm{CO}_{2}, \mathrm{SO}_{2}$ and $\mathrm{NO}_{2}$. These sensors consume little power and are very accurate. GSM modules are used for the wireless communication between the base station and remote sensor node. The GSM modules communicate over cellular networks, which means they can communicate over long distances. A MCU is used to control all the processes on the sensor node. The MCU samples the sensor outputs using an internal ADC; it then calculates the gas concentrations and transmits the computed data as packets using the GSM. A test incubator was designed and constructed to evaluate the performance of the sensor node. The incubator is simply a rectangular plastic box with a hole on the side used to pump gas into it from gas cylinders. The sensor node is tested by placing it inside the incubator; pumping gas into the incubator and observing the measurements taken by the sensor node.

The base station comprises a sink node serially connected to a computer which runs the GUI software. The sink or receiving node captures the data transmitted by the remote sensor node and serially forwards it to the computer. The data is then plotted on the GUI and stored in text files. The items proposed for future work are summarised as follows: energy harvesting could be done to provide power to the sensor nodes [45]-[47]; a solar panel can be used to recharge the batteries powering the sensor node (We will also consider the recharge opportunities based on harvesting techniques. Energy harvesting for sensor modules have the potential to address the design goals of lifetime and performance and make the sensor modules self-sustainable in terms of electrical power supply); a mobile app can be developed to allow users to receive gas concentration measurements on their phones; the detection of gases at lower concentrations (ppb range) can also be done by using more sensitive and more reactive sensors; a control system method using feedback can be used to make the sensors selfcalibrating. Also, the real-time data storage on a mobile server can be implemented where clients can check the level of greenhouse gas concentrations and corresponding locations.

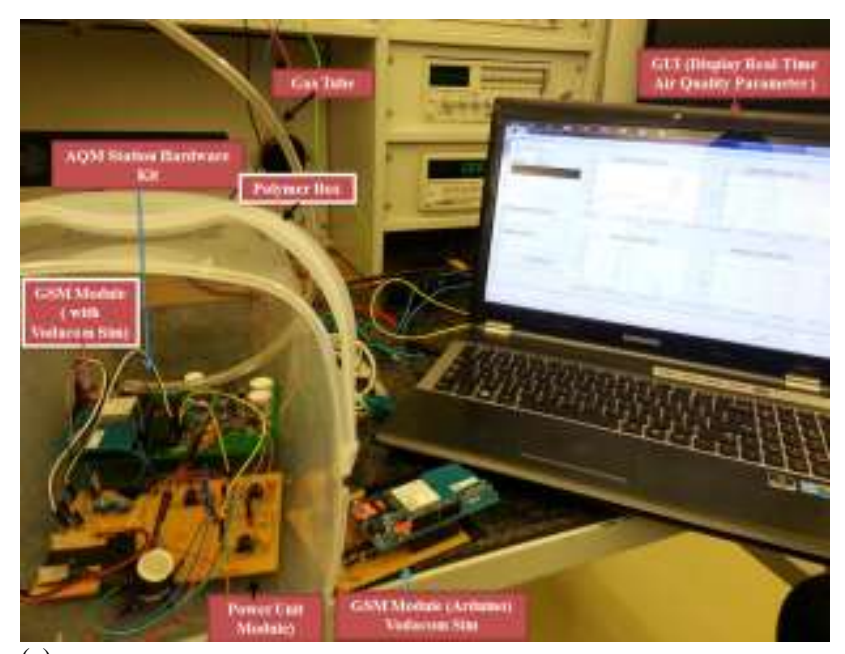

(a)

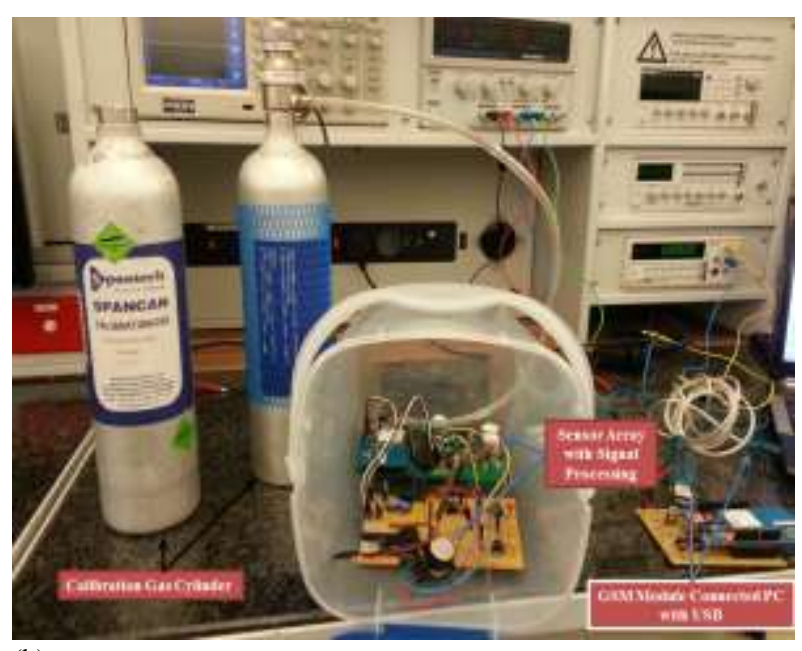

(b)

Fig. 8 (a \& b). Calibration setup and control cross sensitivity or interfering gases setup.

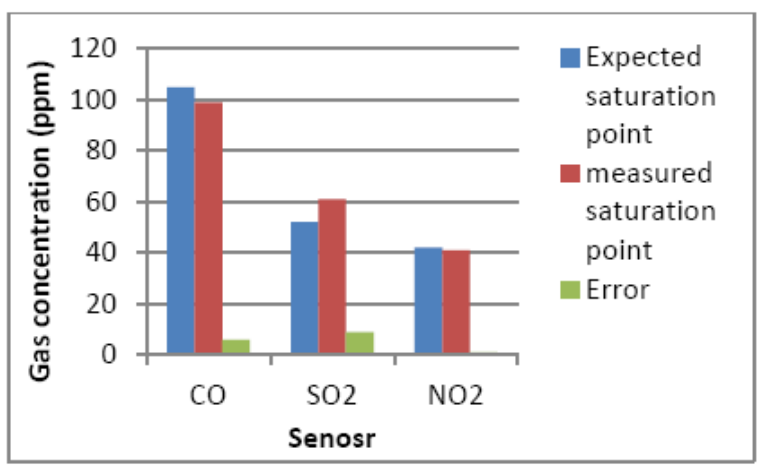

Fig. 9. Comparison of performance of the electrochemical sensors.

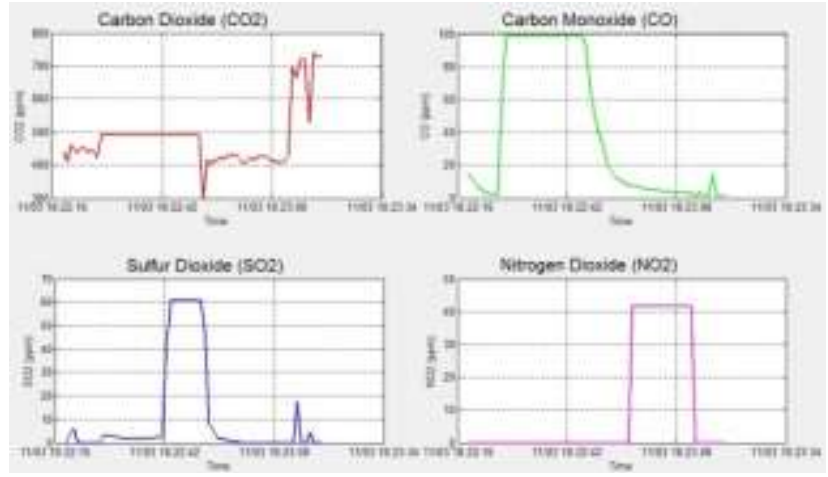

Fig. 10. On-line data updating on GUI based on Real-time measurements of greenhouse gases. 


\section{REFERENCES}

[1]. A Kumar, H Kim and GP Hancke, "Environmental Monitoring Systems: A Review," IEEE Sensors J., vol. 13, no. 4, pp. 1329 - 1339, Apr 2013.

[2]. Report on "Advancing the science of climate change", National Research Council. The National Academies Press, Washington, DC, USA, (2010)

[3]. R.A. Houghton, "Carbon Flux to the Atmosphere from Land-Use Changes", In TRENDS: A Compendium of Data on Global Change. Carbon Dioxide Information Analysis Center, Oak Ridge National Laboratory, U.S. Department of Energy, Oak Ridge, Tenn., U.S.A, 2008.

[4]. R Fisher, L Ledwaba, G Hancke and C Kruger, "Open Hardware: A Role to Play in Wireless Sensor Networks?” Sensors, vol. 15, no 3, pp. 6818-6844, 2015.

[5]. Z Sun, I Akyildiz, and GP Hancke, "Dynamic Connectivity in Wireless Underground Sensor Networks," IEEE Transactions on Wireless Communications," vol. 10, no. 12, 4334-4344, 2011.

[6]. VC Gungor and GP Hancke, "Industrial Wireless Sensor Networks: design principles, and technical approach," IEEE Trans. on Industrial Electronics, vol. 56, no. 10, pp. 4258-4265, Oct. 2009.

[7]. A. Kumar, V. Srivastava, M. K. Singh, and G. P. Hancke, "Current status of the IEEE 1451 standard based sensor applications," IEEE Sensors J., vol. 15, no. 5, pp. 2505-2513, May 2015.

[8]. M. C. Rodriguez-Sanchez, S. Borromeo, and J. A. HernandezTamames, "Wireless sensor networks for conservation and monitoring cultural assets," IEEE Sensors J., vol. 11, no. 6, pp. 1382-1389, June 2011.

[9]. A. Kumar, I. P. Singh, and S. K. Sud, "Energy efficient and low cost indoor environment monitoring system based on the IEEE 1451 standard," IEEE Sensors J., vol. 11, no. 10, pp. 2598-2610, Oct. 2011.

[10]. N. Kularatna and B. H. Sudantha, "An environmental air pollution monitoring system based on the IEEE 1451 standard for low cost requirements," IEEE Sensors J., vol. 8, no. 4, pp. 415 - 422, Apr. 2008.

[11]. A. Kumar and G. P. Hancke, "Energy efficient environment monitoring system based on the IEEE 802.15.4 standard for low cost requirements", IEEE Sensors J., vol. 14, no. 8, pp. 2557-2566, Aug. 2014.

[12]. S. C. Folea and G. Mois, "A low-power wireless sensor for online ambient monitoring," IEEE Sensors J., vol. 15, No.24, pp. 742-749, Feb. 2015.

[13]. F. Sarry and M. Lumbresas, "Gas discrimination in an air-conditioned system," IEEE Trans. Instrum. Meas., vol. 59, no. 4, pp. 809-812, Aug. 2000.

[14]. V. Jelicic, M. Magno, D. Brunelli, G. Paci, and L. Benini, “Contextadaptive multimodal wireless sensor network for energy-efficient gas monitoring," IEEE Sensors J., vol. 13, no. 1, pp. 328-338, Jan. 2013.

[15]. C. Chen, F. Tsow, K. D. Campbell, R. Iglesias, E. Forzani, and N. Tao, "A wireless hybrid chemical sensor for detection of environmental volatile organic compounds," IEEE Sensors J., vol. 13, no. 5, pp. 1748-1755, May 2013.

[16]. W. Chung and J. O. Sung, "Remote monitoring system with wireless sensors module for room environment," Sensors and Actuators B, vol. 113, no. 1, pp. 67-70, Jan. 2006.

[17]. Y. C. Kan, S. Y. Chiang, and C. J. Lin, "A GPS anchor node for outdoor wireless sensor network applications," in Proc. IEEE Int. Symp. Radio-Frequency Integration Technology, Singapore, 2009, pp. 9-11.

[18]. A Kumar and GP Hancke, "An energy-efficient smart comfort sensing system based on the IEEE 1451 standard for green buildings," IEEE Sensors J, vol. 14, no. 12, pp. 4245-4252, Dec. 2014.

[19]. A. Lay-Ekuakille, S. Gioffreda, and A. Trotta, "A smart-autolearned architecture and protocol fo volatile organic compound measurement," in Proc. XVIII Imeko World Congr., Rio de Janeiro, Brazil, Sep. 17-22, 2006.

[20]. R. Yan, D. Ball, A. Deshmukh, and R. Gao, "A Bayesian network approach to energy-aware distributed sensing," in Proc. IEEE Sens. Conf., Vienna, Austria, Oct. 2004, pp. 44-47.

[21]. A. R. Ali, I. Zualkernan, and Fadi Aloul, "A mobile GPRS-sensors array for air pollution monitoring," IEEE Sensors J., vol. 10, no. 10, pp. 1666-11671, Oct. 2010.

[22]. F. Tsow, E. Forzani, A. Rai, R. Wang, R. Tsui, S. Mastroianni, C. Knobbe, A. J. Gandolfi, and N. J. Tao, "A wearable and wireless sensor system for real-time monitoring of toxic environmental volatile organic compounds," IEEE Sensor Journal, vol. 9, no. 12, Dec. 2009.
[23]. C. Peng, K. Qian, and C. Wang, "Design and application of a VOCmonitoring system based on a ZigBee wireless sensor network," IEEE Sensors J., vol. 15, no. 4, pp. 2555-2268, April 2015.

[24]. R. Mukaro and X. F. Carelse, "A microcontroller based data acquisition system for solar radiation and environmental monitoring," IEEE Trans. Instrum. Meas., vol. 48, no. 6, pp. 1232-1238, Dec. 1999

[25]. ISO/IEC/IEEE Information Technology-Smart Transducer Interface for Sensors and Actuators-Part 2: Transducer to Microprocessor Communication Protocols and Transducer Electronic Data Sheet (TEDS) Formats, ISO/IEC/IEEE Standard 21451-2:2010(E), May 2010..

[26]. J. Y. Kim, C. H. Chu, and S. M. Shin, "ISSAQ: an integrated sensing system for real-time indoor air quality monitoring," IEEE Sensors J., vol. 14, no. 12, pp. 4230-4244, Feb. 2014.

[27]. SGX SensorTech sensors data sheet, "designing electronics for infrared sensors", AN4 version 2, England, SGX SensorTech (IS) LTD. Available [online]: http://www.sgxsensortech.com/content/uploads/2014/08/AN4\%E2\%80\%93-Design-of-Electronics-for-Infrared-Gas-Sensors.pdf.

[28]. dsPIC30F3013 Microcontroller datasheet, Microchip Technology Inc., 2010.

[29]. https://www.arduino.cc/en/Main/ArduinoGSMShield.

[30]. I. Stojmenovic, "Machine-to-machine communications with innetwork data aggregation, processing, and actuation for large-scale cyber-physical systems," IEEE Internet of Things Journal, vol. 1, no. 2, pp. 122-128, April 2014.

[31]. K. C. Chen and S. Y. Lien, "Machine-to-machine communications: technologies and challenges," Ad Hoc Networks, vol. 18, pp. 3-23, 2014.

[32]. S. Y. Lien, K. C. Chen, and Y. H. Lin, "Toward ubiquitous massive accesses in 3GPP machine-to-machine communications," IEEE Communications Magazine, vol. 49, no. 4, pp. 66-74, April 2011

[33]. 3GPP TS 22.368 V12.0.0, service requirement for machine-type communications, Sep. 2012

[34]. 3GPP TR 23.888 V11.0.0, system improvement for machine-type communications, Sep. 2012.

[35]. V. Galetic, I. Bojic, M. Kusek, G. Jezic, S. Desic, and D. Huljenic, "Basic principles of machine-to-machine communication and its impact on telecommunications industry," Proceedings of the 34th International Convention MIPRO 2011, 23-27 May, Opatija, Croatia, pp. 380-385.

[36]. M. Dujak, V. Parac, M. Durasevic, and A. Heric, "Machine-tomachine communication as key enabler in smart metering system," Proceedings of the $36^{\text {th }}$ International Convention MIPRO 2011, 20-24 May, Opatija, Croatia, pp. 409-414.

[37]. N. Nikaein and S. Krco, "Latency for real-time machine-to-machine communication in LTE-based system architecture," $11^{\text {th }}$ European Wireless Conference 2011, 27-29 April 2011, Vienna, Austria, pp. 263-268.

[38]. Y. Chen and W. Wang, "Machine-to-machine communication in LTE-A," Proceedings of the $72^{\text {th }}$ IEEE Vehicular Technology Conference 2010 (Fall), VTC 2010-Fall), 6-9 Sept. 2010, pp. 1-5.

[39]. Y. Chen and Y. Yang, "Cellular based machine to machine communication with un-peer2peer protocol stack," Proceedings of the $70^{\text {th }}$ IEEE Vehicular Technology Conference 2009 (Fall), VTC 2009-Fall), 20-23 Sept. 2009, pp. 1-5.

[40]. I. Doh, J. Lim, S. Li, and K. Chae, "Key establishment and management for secure cellular machine-to-machine communication," $7^{\text {th }}$ Inter. Conf. on Innovative Mobile and Internet in Ubiquitous Computing, 3-5 July 2013, Taichung, Tiwan, pp. 579584.

[41]. M. Hasan, E. Hossain, and D. Niyato, "Random access for machineto-machine communication in LTE-advanced networks: issues and approaches," IEEE Communications Magazine, vol. 51, no. 6, pp. 86-93, June 2013.

[42]. H. K Lee, D. M. Kim, Y. J. Hwang, S. M. Yu, and S. L. Kim, "Feasibility of cognitive machine-to-machine communication using cellular bands," IEEE Wireless Communications, vol. 20, no. 2, pp. 97-103, April. 2013.

[43]. C.P. Kruger and G.P. Hancke, "Implementing the Internet of Things vision in industrial wireless sensor networks," IEEE International Conference on Industrial Informatics, pp.627-632, July 2014.

[44]. K. B. Shaban, A. Kadri, and E. Rezk, "Urban air quality monitoring system with forecasting models," IEEE Sensors J., vol. 16, no. 8, pp. 2598-2606, April 2016.

[45]. J.A. van Schalkwyk and G.P. Hancke, "Energy Harvesting for Wireless Sensors from Electromagnetic Fields around Overhead Power Lines", 2012 IEEE International Symposium on Industrial Electronics (ISIE), pp. 1128 - 1135. 
[46]. D. Ramasur and G.P. Hancke, "A wind energy harvester for low power wireless sensor networks", IEEE International Instrumentation and Measurement Technology Conference (I2MTC), 2012 , pp. $2623-2627$.

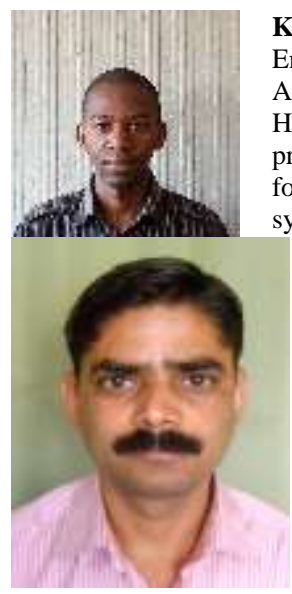

K. S. E. Phala received the BEng in Computer Engineering from the University of Pretoria, South Africa, in 2014, where he is currently pursuing the Honours degree in Computer Engineering, with his primary research area being the Internet of Sensors for industrial applications. This includes embedded systems, wireless sensor networks, sensor arrays, intelligent buildings and mobile computing. Since February 2015 he has been employed as an Engineer at ArcelorMittal.

Anuj Kumar received his M. Tech. degree in Instrumentation from the N.I.T. Kurukshetra, India in 2004, and Ph.D. degree in Electrical Engineering from the I.I.T. Delhi, India in 2011.

He is currently as a Ramanujan Fellow, in CSIR-CBRI Roorkee, India. His research interests include, wireless sensor actuators networks, and pollution measurement for industry. He has carried out extensive research in smart building areas of the development of hardware and software for experimental prototypes

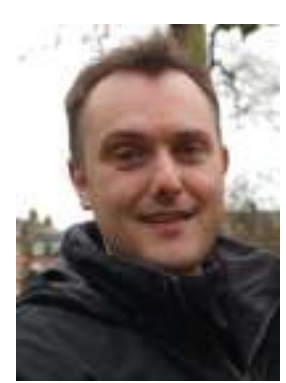

Gerhard P. Hancke is an Assistant Professor in the Department of Computer Science at City University of Hong Kong. He obtained B.Eng. and M. Eng. degrees from the University of Pretoria (South Africa) and a PhD in Computer Science from the University of Cambridge in 2008. He is interested in sensing systems for industrial applications. He is also Extraordinary Senior Lecturer at University of Pretoria.
[47]. B. Silva, R. Fisher, A. Kumar, and G. Hancke, "Experimental Link Quality Characterization of Wireless Sensor Networks for Underground Monitoring," IEEE Transactions on Industrial Informatics, vol. 11, no. 5, pp. 1099-1110, Oct. 2015. 